\title{
Consensus of Two Leader-Following Multi-agent Systems between the Cooperation Network and the Competition Network
}

\author{
Miao Liu* \\ College of Electronic and Information Engineering, Southwest University, Chongqing 400715, China. \\ * Corresponding author. Email: lmhappy@email.swu.edu.cn \\ Manuscript submitted January 10, 2016; accepted May 8, 2016. \\ doi: $10.17706 /$ jcp.12.5.389-395
}

\begin{abstract}
In this paper, a cooperation network and a competition network consensus problem of multi-agent systems is study. The communication topology among agent is characterized by a directed and connected graph. And Communication between the cooperation network and the competition network through their respective leaders. The two networks can be made to reach stable and consensus by setting parameters and between the two leaders. Sufficient conditions are derived to reach a global consensus based on algebraic graph , matrix theory, and stability theory. With theoretical analysis, it obtains the value range of the parameters. For the case of switching topology, a sufficient condition is obtained under the assumption that the total period over which the leader is globally reachable is sufficiently large. Finally, through the MATLAB simulation, an example is presented to illustrate the correctness of the theoretical results.
\end{abstract}

Key words: Cooperation and competition, leader-following, consensus.

\section{Introduction}

In recent years, great interest has been aroused in research on multi-agent systems (MAS) [1]-[6], whose consensus problems also attract a lot of attentions of researchers. To a great extent, this is because their wide applications in flocking, formation control about unmanned vehicles and congestion control in communication networks [7]-[12].

As a very valuable important issue in the multi- agent systems, consensus means agents achieving an agreement on their common state by using information from their local neighbors. Consensus can not only be used to explain flocking of social animals but also can be applied on attitude alignment, air traffic control, wireless sensor networks and mobile Robotic swarms [13]-[16]. Consensus problems of multi-agent systems are often refined into higher-order consensus, finite-time consensus, adaptive consensus, heterogeneous consensus and leader-following consensus [17]-[25].

Leader-following consensus is a major theoretical and practical value problems, which is widely used in the fields of unmanned air vehicles, mobile robotic swarms, wireless sensor networks and cooperative surveillance [26]-[29]. For the case of leader-following topologies, in [30], the leader-following consensus for multi-agent systems was studied by using the sampled-data control approach. The topology considered is deterministic. Both leaderless and leader-following consensus problems were studied in [31]. And through using Lyapunov theorems and Nyquist stability criterion, the stability or bounded-ness conditions were presented. In [32], the leader-following consensus problem of multi-agent systems with double-integrator 
dynamics was considered, and then a necessary and sufficient condition for the case of fixed topologies and a sufficient condition for the case of switching topologies were shown. In [33], distributed observers were designed through learning from a leader-following consensus problem for a multi-agent system with a switching topology.

However, almost all of the previous studies were only concerned with the consensus of multi-agent systems or Leader-following consensus. This paper focuses on studying the consensus problem of both multi-agent systems and Leader-following.

This paper is organized as follows. First, two multi-agent systems with leader between the cooperation network and the competition network is designed. Then, stability of sufficient condition of two multi-agent systems is obtained. Finally, numerical simulations are implemented to demonstrate the analytic results.

\section{Topology Description}

In this section, some basic definition in graph theory, the system model, are firstly introduced for subsequent use. Graphs are used to describe the interconnected topology of agents.

We consider two system, and one consists of $N$ agents and a leader (labeled by 0 ) which is depicted by a graph $G$, and the other also consists of $N$ agents and a leader (labeled by $0^{\prime}$ ) which is depicted by a graph $S$. Let $G=(V, \zeta, A)$ be a weighted digraph with a set of nodes $V=\{1,2, \ldots, N\}$, a set of edges $\zeta \subseteq V \times V$ and a weighted adjacency matrix $A=\left[a_{i j}\right] \in R^{N \times N}$ with nonnegative elements. A directed edge $(j, i) \in \zeta$ denotes that agent $i$ can obtain information from agent $j$, or agent $j$ can reach agent $i$. If there is an edge from agent $j$ to agent $i$, agent $j$ is called a neighbor of agent $i$ and $a_{i j}>0$; otherwise $a_{i j}=0$. The set of neighbors of the $i$ th agent $i$ is denoted by $N_{i}=\{j \in V \mid(j, i) \in \zeta\}$. Diagonal matrix $D=\operatorname{diag}\left\{d_{1}, d_{2}, \ldots, d_{N}\right\}$, whose diagonal elements $d_{i}=\sum_{j \in N_{i}} a_{i j}$, is the degree matrix.At the same time, let $S=(\pi, \varepsilon, C)$ be a weighted digraph with a set of nodes $\pi=\{1,2, \ldots, N\}$, a set of edges $\varepsilon \subseteq \pi \times \pi$ and a weighted adjacency matrix $C=\left[c_{i j}\right] \in R^{N \times N}$ with nonnegative elements.

The Laplacian of weighted digraph $G$ and weighted digraph $S$ is defined as $L_{G}=D-A$ and $L_{S}=F-C$, respectively. Diagonal matrix $B=\operatorname{diag}\left\{b_{1}, b_{2}, \ldots, b_{N}\right\}$ and diagonal matrix $E=\operatorname{diag}\left\{e_{1}, e_{1}, \ldots, e_{N}\right\}$ are the leader adjacency matrix associated with $G$ and $S$, where $b_{i}>0$ and $e_{i}>0$ if nodes are the neighbor of node $i, b_{i}=0$ and $e_{i}=0$ otherwise. $\otimes$ represents the Kronecker product of matrices.

\section{Problem Formulation}

In this paper some mathematical natation will be presented as follow. $R^{n}$ denote the n-dimensional real vector space. Consider system (1) and (2) withs witching graphs. The system (1) corresponding Laplacian, degree matrix, adjacency matrix and leader adjacency matrix are denoted by $L_{G}, D, A$ and $B$, respectively, and $H_{G}=L_{G}+B$. The system (2) corresponding Laplacian, degree matrix, adjacency matrix and leader adjacency matrix are denoted by $L_{S}, F, C$ and $E$, respectively, and $H_{S}=L_{S}+C$.

Consider the following two systems, one is cooperation network and the competition network with leader. Two networks only through their own leader communication (cooperation-competition), $\alpha, \beta$ is the mutual influence between the two leader. By setting the topology para-meters to make the model to be uniformly stable. Therefore, we propose the following framework described by (1) and (2): 


$$
\begin{aligned}
& \left\{\begin{array}{l}
\dot{x}_{i}(t)=-\sum_{j=1}^{N} a_{i j}\left(x_{i}(t)-x_{j}(t)\right)-b_{i}\left(x_{i}(t)-x_{0}(t)\right) \\
\dot{x}_{0}(t)=\sum_{j=1}^{N} b_{i}\left(x_{i}(t)-x_{0}(t)\right)-\alpha\left(x_{0}(t)-y_{0}(t)\right)
\end{array}, i=1,2, \ldots, N\right. \\
& \left\{\begin{array}{l}
\dot{y}_{i}(t)=-\sum_{j=1}^{N} c_{i j}\left(y_{i}(t)-y_{j}(t)\right)-e_{i}\left(y_{i}(t)-y_{0}(t)\right) \\
\dot{y}_{0}(t)=\sum_{j=1}^{N} e_{i}\left(y_{i}(t)-y_{0}(t)\right)+\beta\left(x_{0}(t)-y_{0}(t)\right)
\end{array}, i=1,2, \ldots, N\right.
\end{aligned}
$$

where $\alpha, \beta \in R$ is the mutual influence parameters between the two leader.

Considered as a whole, between four dynamic system is coupled to each other,

$$
\left\{\begin{array}{l}
\dot{x}_{i}(t)=-\sum_{j=1}^{N} a_{i j}\left(x_{i}(t)-x_{j}(t)\right)-b_{i}\left(x_{i}(t)-x_{0}(t)\right) \\
\dot{x}_{0}(t)=\sum_{i=1}^{N} b_{i}\left(x_{i}(t)-x_{0}(t)\right)-\alpha\left(x_{0}(t)-y_{0}(t)\right) \\
\dot{y}_{i}(t)=-\sum_{j=1}^{N} c_{i j}\left(y_{i}(t)-y_{j}(t)\right)-e_{i}\left(y_{i}(t)-y_{0}(t)\right) \\
\dot{y}_{0}(t)=\sum_{i=1}^{N} e_{i}\left(y_{i}(t)-y_{0}(t)\right)+\beta\left(x_{0}(t)-y_{0}(t)\right)
\end{array}\right.
$$

Then system (3) can be simplified as follows.

$$
\left\{\begin{array}{l}
\dot{x}(t)=-H_{a} x(t)+b x_{0}(t) \\
\dot{x}_{0}(t)=\left(-\sum_{i=1}^{N} b_{i}-\alpha\right) x_{0}(t)+b^{T} x(t)+\alpha y_{0}(t) \\
\dot{y}(t)=-H_{c} y(t)+e y_{0}(t) \\
\dot{y}_{0}(t)=\left(-\sum_{i=1}^{N} e_{i}-\beta\right) y_{0}(t)+e^{T} y(t)+\beta x_{0}(t)
\end{array}\right.
$$

Let $\varepsilon(t)=\left[\begin{array}{l}x(t) \\ x_{0}(t) \\ y(t) \\ y_{0}(t)\end{array}\right]$, The equation (4) can be translated into: $\dot{\varepsilon}(t)=A^{\prime} \varepsilon(t)$

where $A^{\prime}=\left[\begin{array}{cccc}-H_{a} & b & 0 & 0 \\ b^{T} & -\sum_{i=1}^{N} b_{i}-\alpha & 0 & \alpha \\ 0 & 0 & -H_{c} & e \\ 0 & \beta & e^{T} & -\sum_{i=1}^{N} e_{i}-\beta\end{array}\right]$

Theorem 1: Suppose that $H_{G}$ and $H_{S}$ contain the directed spanning tree, then systems (1) and (2) is uniformly stable if $\alpha>-\sum_{i=1}^{N} b_{i}+b^{T}\left(H_{G}\right)^{-1} b$ and $\left|\left[\begin{array}{cc}-H_{S} & e \\ e^{T} & -\sum_{i=1}^{N} e_{i}-\beta\end{array}\right]-\left[\begin{array}{ll}0 & 0 \\ 0 & \beta\end{array}\right]\left[\begin{array}{cc}-H_{G} & b \\ b^{T} & -\sum_{i=1}^{N} b_{i}-\alpha\end{array}\right]^{-1}\left[\begin{array}{ll}0 & 0 \\ 0 & \alpha\end{array}\right]\right|>0$ 
Proof. Just the eigenvalues of $A$ is the negative.

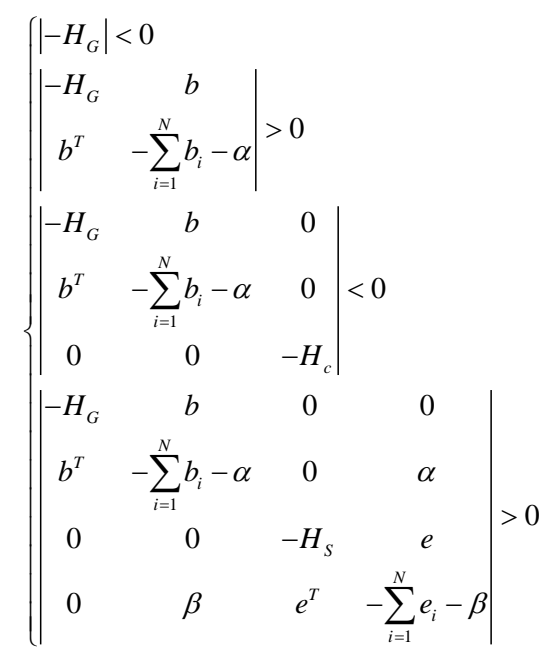

Suppose that $H_{G}$ and $H_{S}$ contain the directed spanning tree, so $\left|-H_{G}\right|<0$. Then by (5), the following sufficient condition can be obtained:

$$
\alpha>-\sum_{i=1}^{N} b_{i}+b^{T}\left(H_{G}\right)^{-1} b \text { and }\left|\left[\begin{array}{cc}
-H_{S} & e \\
e^{T} & -\sum_{i=1}^{N} e_{i}-\beta
\end{array}\right]-\left[\begin{array}{cc}
0 & 0 \\
0 & \beta
\end{array}\right]\left[\begin{array}{cc}
-H_{G} & b \\
b^{T} & -\sum_{i=1}^{N} b_{i}-\alpha
\end{array}\right]^{-1}\left[\begin{array}{ll}
0 & 0 \\
0 & \alpha
\end{array}\right]\right|>0
$$

Remark 1. Compared with [34], we consider a cooperation network and a competition network consensus problem of multi-agent systems.

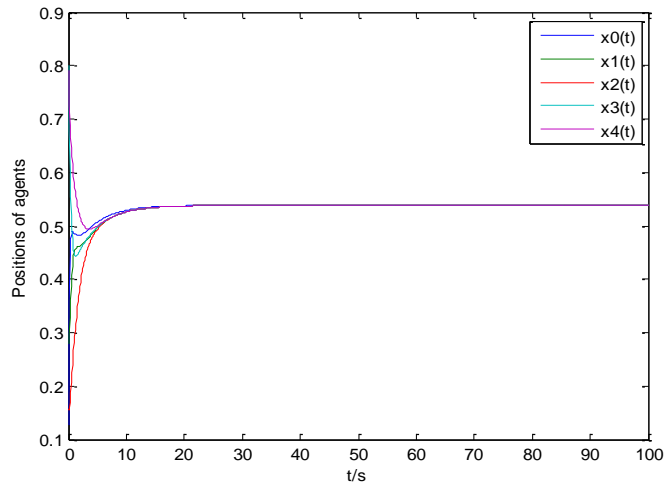

Fig. 1. The evolution of states $x(t)$.

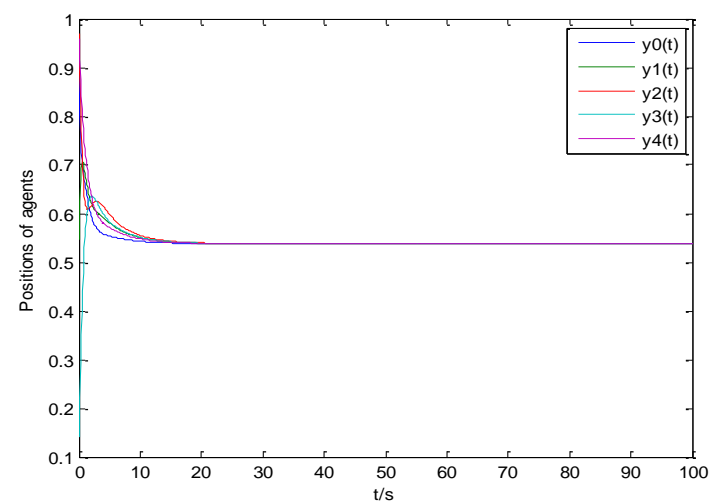

Fig. 2. The evolution of states $y(t)$.

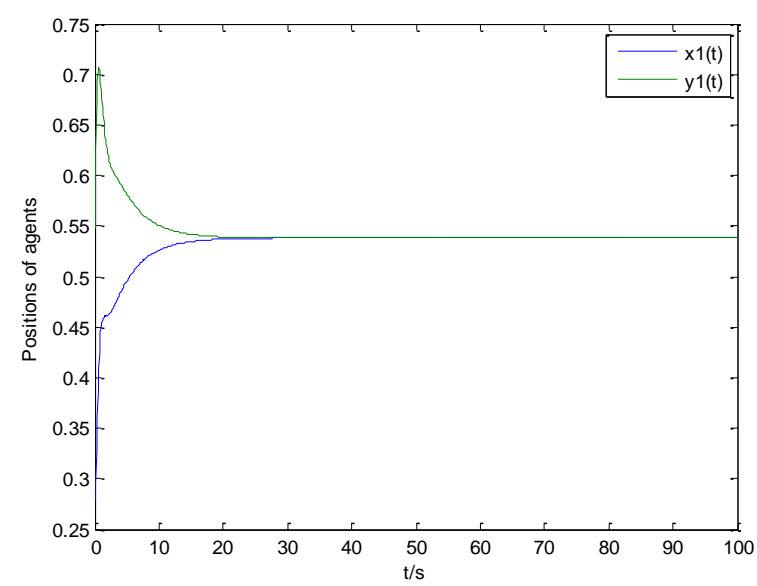

Fig. 3. The evolution of states $x(t)$ and $y(t)$. 


\section{Numerical Simulation}

In this section, one simulation experiment are provided to demonstrate the feasibility and effectiveness

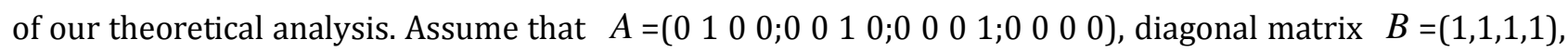

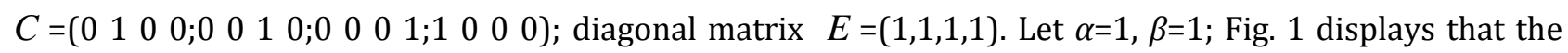
multi-agent network (1) is the evolution of states $x(t)$. Fig. 2 shows the evolution of states $y(t)$. And meanwhile, Fig. 3 demonstrates the evolution of states $x(t)$ and $y(t)$.

\section{Conclusion}

This paper discussed the consensus of two multi-agent systems with leader between the cooperation network and the competition network. We get a sufficient condition when two multi-agent networks achieve consensus. Namely, the range of parameters $\alpha, \beta$. We assume that the multi-agent network is strongly connected or contains a directed spanning tree. Network-based consensus is studied in the consensus of two multi-agent systems with leader between the cooperation network and the competition network.

\section{Conclusion}

A conclusion section is not required. Although a conclusion may review the main points of the paper, do not replicate the abstract as the conclusion. A conclusion might elaborate on the importance of the work or suggest applications and extensions.

\section{Acknowledgment}

This work described in this paper was supported in part by the National Natural Science Foundation of China under Grant nos.61170249.

\section{References}

[1] Ren, W., Beard, R. W., \& Atkins, E. M. (2005). A survey of consensus problems in multiagent coordination. Proceeding of the 2005 American Control Conference (pp. 1859-1864).

[2] Lin, Z., Francis, B., \& Maggiore, M. (2008). Distributed Control and Analysis of Coupled Cell Systems, VDM Publishing.

[3] Zheng, Y., Zhu, Y., \& Wang, L. (2011). Consensus of heterogeneous multi-agent systems. IET Control Theory Appl., 5(16), 1881-1888.

[4] Olfati-Saber, R., Fax, J. A., \& Murray, R. M. (2007). Consensus and cooperation in networked multi-agent systems. Proceedings of IEEE, 95(1), 215-233.

[5] Xiao, F., Wang, L., \& Chen, T. (2011). Finite-time consensus of multi-agent systems with directed and intermittent links. Proceedings of the 30st Chinese Control Conference (pp. 6539-6543).

[6] Zhong, M., \& Cassandras, C. (2010). Asynchronous distributed optimization with event-driven communication. IEEE Trans. Automat. Control, 55(12), 2735-2750.

[7] Fax, A., \& Murray, R. (2004). Information flow and cooperative control of vehicle formations. IEEE Transactions on Automatic Control, 49, 1453-1464.

[8] Tang, Y., Wang, Z. \& Fang, J. (2011). Controller design for synchronization of an array of delayed neural networks using a controllable probabilistic PSO. Inf. Sci., 181, 4715-4732.

[9] Xiao, F., Wang, L., Chen, J., \& Gao, Y. (2009). Finite-time formation control for multi-agent systems. Automatica, 45(11), 2605-2611.

[10] Olfati-Saber, R. (2006). Flocking for multi-agent dynamic systems: algorithms and theory. IEEE Trans. Automat. Control, 51(3), 401-420.

[11] Tanner, H., Jadbabaie, A., \& Pappas, G. (2007). Flocking in fixed and switching networks. IEEE Trans. 
Automat. Control, 52 (5), 863-868.

[12] Liu, Y., Slotine, J. J., \& Barabasi. A. L. (2011). Controllability of complex networks. Nature, 473(7346), 167-173.

[13] Wang, P. K. C., \& Hadaegh, F. Y. (1996). Coordination and control of multiple microspacecraft moving in formation. The Journal of the Astronautical Sciences, 44(3), 315-355.

[14] Meng, Z., Ren, W., \& You, Z. (2010). Distributed finite-time attitude containment control for multiple rigid bodies. Automatica, 46(12), 2092-2099.

[15] Wang, Y., \& Cao, J. (2013). Cluster synchronization in nonlinearly coupled delayed networks of non-identical dynamic systems. Nonlinear Anal. Real, 14(1).

[16] Yu, W., Chen, G., Wang, Z., \& Yang, W. (2009). Distributed consensus filters in sensor networks. IEEE Trans. Syst, Man, Cybern. B, Cybern., 39(6), 1568-1577.

[17] Jiang, F., \& Wang, L. (2011). Finite-time weighted average consensus with respect to a monotonic function and its application. Syst. Control Lett., 60(9), 718-725.

[18] Franceschelli, M., Giua, A., Pisano, A., \& Usai, E. (2013). Finite-time consensus for switching network topologies with disturbances. Nonlinear Anal. Hybrid, 10, 83-93.

[19] Xiao, F., Wang, L., Chen, J., \& Gao, Y. (2009). Finite-time formation control for multi-agent systems. Automatica, 45(11), 2605-2611.

[20] Ren, W., Moore, K. L., \& Chen, Y. (2007). High-order and model reference consensus algorithms in cooperative control of multi-vehicle systems. ASME J. Dyn. Syst. Meas. Control, 129(5), 678-688.

[21] Jiang, F., \& Wang, L. (2010). Consensus seeking of high-order dynamic multi-agent systems with fixed and switching topologies. Int. J. Control, 85(2), 404-420.

[22] Shao, J., Xie, G., \& Wang, L. (2007). Leader-following formation control of multiple mobile vehicles. IET Control Theory, A1(2), 545-552.

[23] Ding, Z. (2013). Consensus output regulation of a class of heterogeneous nonlinear systems. IEEE Trans. Autom. Control, 58(10), 2648-2653.

[24] Zheng, Y., \& Wang, L. (2012). Finite-time consensus of heterogeneous multi-agent systems with and without velocity measurements. Syst. Control Lett., 61(8), 871-878.

[25] Yang, X., Cao, J., Long, Y., \& Rui, W. (2010). Adaptive lag synchronization for competitive neural networks with mixed delays and uncertain hybrid perturbations. IEEE Trans. Neural Netw., 21(10), 1656-1667.

[26] Wei, R. (2007). Multi-vehicle consensus with a time-varying reference state. Syst ControlLett, 56(7-8), 474-483.

[27] Wei, R. (2010). Consensus tracking under directed interaction topologies: Algorithms and experiments. IEEE Trans. Control Syst. Technol., 18(1), 230-237.

[28] Jie, M., Wei, R., \& Ma, G. F. (2011). Distributed coordinated tracking with a dynamic leader for multiple Euler-Lagrange systems. IEEE Trans. Autom Control, 56(6), 1415-1421.

[29] Wen, G. G., Peng, Z. X., Ahmed, R., Yu, Y. G. (2014). Distributed leader-following consensus for second-order multi-agent systems with nonlinear inherent dynamics. Int. J. Syst. Sci., 45(9).

[30] Tang, Z.-J., Huang, T.-Z., Shao, J.-L., \& Hu, J.-H. (2011). Leader-following consensus for multi-agent systems via sampled-data control. IET Control Theory Appl., 5(14), 1658-1665.

[31] Meng, Z. Y., Wei, R., Cao, Y. C., \& You, Z. (2011). Leaderless and leader following consensus with communication and input delays under a directed network topology. IEEE Trans. Syst. ManCybern.-Part B: Cybern., 41(1), 75-88.

[32] Zhu, W., \& Cheng, D. (2010). Leader-following consensus of second-order agents with multiple time-varying delays. Automatica, 46(12), 1994-1999. 
[33] Hong, Y., Chen, G., \& Bushnell, L. (2008). Distributed observers design for leader-following control of multi-agent networks. Automatica, 44(3), 846-850.

[34] Hu, H. X., Yu, W., Xuan, Q., Yu, L., \& Xie, G. (2015). Consensus of multi-agent systems in the cooperation-competition network with inherent nonlinear dynamics: A time-delayed control approach. Neurocomputing, 158, 134-143.

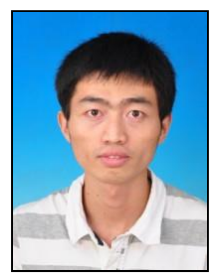

Liu Miao was born in Heilongjiang, China, in 1988. Currently, he is working towards the M.Sc. degree at the College of Electronic and Information Engineering, Southwest University, Chongqing, China from September 2013 to June 2016. His current research interests include nonlinear dynamical systems, multi-agent systems, complex networks, and switched systems. 